\title{
Special Section on Ethics in Health Informatics
}

\author{
Carolyn Petersen', Vignesh Subbian'2, Section Editors Special Section on Ethics in Health \\ Informatics of the International Medical Informatics Association Yearbook \\ 1 Division of Biomedical Statistics and Informatics, Mayo Clinic, Rochester, Minnesota, USA \\ ${ }^{2}$ College of Engineering, The University of Arizona, Tucson, Arizona, USA
}

\begin{abstract}
Summary
Objective: To summarize significant research contributions on ethics in medical informatics published in 2019.

Methods: An extensive search using PubMed/Medline was conducted to identify the scientific contributions published in 2019 that address ethics issues in medical informatics. The selection process comprised three steps: 1) 15 candidate best papers were first selected by the two section editors; 2) external reviewers from internationally renowned research teams reviewed each candidate best paper; and 3) the final selection of three best papers was conducted by the editorial committee of the Yearbook. Results: The three selected best papers explore timely issues of concern to the community and demonstrate how ethics considerations influence applied informatics.

Conclusion: With regard to ethics in informatics, data sharing and privacy remain primary areas of concern. Ethics issues related to the development and implementation of artificial intelligence is an emerging topic of interest.
\end{abstract}

\section{Keywords}

Data sharing, ethics, personal health data, privacy

Yearb Med Inform 2020:77-80

http://dx.doi.org/10.1055/s-0040-1702014

\section{Introduction}

Ethical, Legal, and Social Issues (ELSI) in medical informatics grow in tandem with advances in digital health and computing technologies. The year 2019 was marked by critical debates and scholarship on privacy and data sharing related to personal health. First, the pervasive technology-enabled collection of all forms of data (health records, social media posts, geolocation, online and credit card purchases) from consumers led to proliferation of health care data economies [1] and raised questions about intended use and perhaps misuse of data, particularly by large corporations. Second, the widespread development of artificial intelligence (AI)based applications for healthcare resulted in a push for effective and responsible ways of validating, governing, and regulating such systems [2]. Third, implementation of new regulations such as the General Data Protection Regulation (GDPR) [3,4] and the California Consumer Privacy Act (CCPA) in mid-2018 led to a renewed focus on balancing privacy and sharing of personal data. In addition to these developments, there was continued interest in addressing ELSI in secondary use of data for biomedical research, especially in the context of large consortium-based studies.

\section{Methods}

The literature search was performed on PubMed/Medline in January 2020. Search queries were developed for three publication categories: ethics journals (e.g., Science and Engineering Ethics), informatics journals (e.g., Journal of the American
Medical Informatics Association), and biomedical journals (e.g., BMJ). Both controlled vocabulary terms (e.g., MeSH) and key words were used in the search queries. A manual search was performed on relevant journals not indexed in PubMed (e.g., Applied Clinical Informatics Open). The inclusion criteria were (1) papers published in English language between January 1, 2019 and December 31, 2019, and (2) topic is of relevance to ELSI and medical informatics. One of the section editors performed the search, which yielded a total of 460 references. Of these, 19 articles were selected based on initial screening of title and abstract. Both section editors reviewed the full text of the 19 articles and categorized them into three groups (accept, discuss, and discard) based on their originality, innovativeness, scientific and/or practical impact, and scientific quality. Fifteen articles that both section editors agreed to accept were submitted as candidate best papers.

In accordance with the International Medical Informatics Association (IMIA) Yearbook selection process, the 15 candidate best papers were further evaluated by the two section editors, the chief editor of the section, and by additional external reviewers (at least four reviewers per paper). The section editors identified potential commercial interests in two of the 15 papers, and one of the section editors (Petersen) is a co-author of one of the candidate best papers (Lehmann et al [5]). To avoid bias in the review of the best paper candidates, neither the section editors nor the chief editor of the section reviewed this paper. Three papers were finally selected as best papers for the special section based on reviewer ratings (Table 1). A content summary of the selected best papers can be found in the appendix of this synopsis. 
Petersen et al.

Table 1 Best paper selection of articles for the IMIA Yearbook of Medical Informatics 2020 in the special section 'Ethics in Health Informatics'. The articles are listed in alphabetical order of the first author's surname.

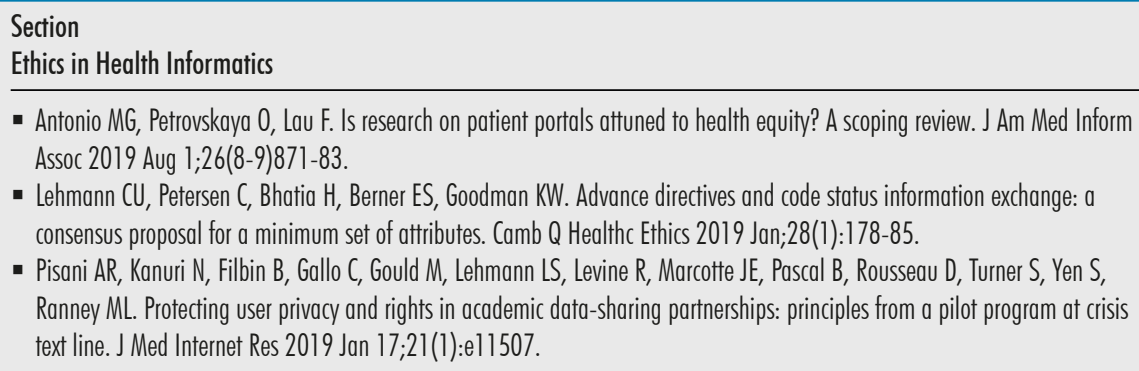

- Lehmann CU, Petersen C, Bhatia H, Berner ES, Goodman KW. Advance directives and code status information exchange: a consensus proposal for a minimum set of attributes. Camb Q Healthc Ethics 2019 Jan;28(1):178-85.

- Pisani AR, Kanuri N, Filbin B, Gallo C, Gould M, Lehmann LS, Levine R, Marcotte JE, Pascal B, Rousseau D, Turner S, Yen S, Ranney ML. Protecting user privacy and rights in academic data-sharing partnerships: principles from a pilot program at crisis text line. J Med Internet Res 2019 Jan 17;21(1):e11507.

\section{Conclusions and Outlook}

Although the number of candidate papers was quite small compared to some other areas of informatics, it is common that few papers in this area meeting the selection criteria are published annually. As is often the case in ethics, the search for a balance between the rights of individuals to retain autonomy and self-determination and the need for society to benefit from initiatives experienced collectively (e.g., healthcare) figured prominently. The continued evolution of patient-centered healthcare and precision medicine, in an environment of rapidly evolving technologies, is creating more opportunities for patients, practitioners, researchers, informaticians, and population health specialists to collaborate and explore new areas. It is unsurprising that robust debate around the appropriate use of personal data and medical knowledge would take place at the forefront of discussion.

In recent years, the majority of papers addressing ethical issues have tended to concentrate on privacy and data sharing. This proved to be the case in 2019. Among the 19 shortlisted papers, seven focused on various forms of data sharing and/or data use, five addressed privacy concerns, and one paper addressed both topic areas. Articles in the data sharing area explored a broad range of issues including passive data collection [6]; [patient] participatory methods in data-intensive biomedical research [7] and disease surveillance [8]; data management, use/re-use, and sharing internationally [9] and under the GDPR [10]; posthumous data donation [11]; and human protection with regard to data sharing [12]. Papers focused on privacy looked at the use and understanding of anonymization and de-identification practices in the literature [13]; health information disclosure [14]; balancing privacy and data use under the GDPR [15]; activities that work against citizen and patient trust with regard to personal information [16]; and terms of use violations by researchers accessing online patient information [17]. The paper addressing both data sharing and privacy, which was selected as a best paper of 2019, proposed guidelines for protection of user privacy and rights in academic data sharing partnerships through the analysis of a crisis text line pilot program [18].

Though data sharing and privacy dominated the results, the search for papers related to ELSI uncovered an emerging area of concern: the appropriate use and governance of artificial intelligence (AI) in informatics. Four papers addressed various aspects of ethics in AI, including the appropriate development, validation, and implementation of AI in patient care [19]; a proposed governance model for AI [20]; support for citizen trust when AI is in use [21]; and ethical issues related to the use of AI in psychiatry [22]. The ongoing development of methods for reliably using large, multimodal data sources, and the need for answers to clinical questions more quickly (e.g., successful treatments for particular conditions) ensure that AI will grow as a focus area for ethics within informatics. Should health care organizations around the world work together to solve shared challenges, such as the effective management of COVID-19 infection, concerns about the appropriate use and management of AI may prove to be the next Grand Challenge for ethics in informatics.

The search for best papers yielded papers on two additional topics, the role of health equity in research on patient portals [23] and considerations related to managing advance directives through the electronic health record [5], both of which were selected as best papers of 2019. The former found that the burden of addressing barriers to portal use often are placed on patients who are already experiencing health inequities. The latter developed a minimum set of data elements for advance directives and code status information exchange among health systems.

\section{Acknowledgement}

We would like to acknowledge the support of Adrien Ugon, Martina Hutter, Kate Fultz Hollis, Lina Soualmia, Brigitte Séroussi, and the Yearbook editorial committee as well as the reviewers for their contribution to the selection process.

\section{References}

1. Perakslis E, Coravos A. Is health-care data the new blood? Lancet Digit Heal 2019;1(1):e8-e9.

2. Anderson M, Anderson SL. How should AI Be developed,validated and implemented in patient care? AMA J Ethics 2019;21(2):125-30.

3. The European Parliament and the Council of the European Union. REGULATION (EU) 2016/679 OF THE EUROPEAN PARLIAMENT AND OF THE COUNCIL of 27 April 2016 on the protection of natural persons with regard to the processing of personal data and on the free movement of such data, and repealing Directive 95/46/EC (General Data Protection Regulation) (Text with EEA relevance); 2016.

4. Rothstein MA, Tovino SA. California Takes the Lead on Data Privacy Law. Hastings Cent Rep 2019;49(5):4-5.

5. Lehmann CU, Petersen C, Bhatia H, Berner ES, Goodman KW. Advance Directives and Code Status Information Exchange: A Consensus Proposal for a Minimum Set of Attributes. Cambridge Q Healthc ethics 2019;28(1):178-85.

6. Maher NA, Senders JT, Hulsbergen AFC, Lamba N, Parker M, Onnela J-P, et al. Passive data collection and use in healthcare: A systematic review of ethical issues. Int J Med Inform 2019;129:242-7.

7. Beier K, Schweda M, Schicktanz S. Taking patient involvement seriously: a critical ethical analysis 
of participatory approaches in data-intensive medical research. BMC Med Inform Decis Mak 2019;19(1):90.

8. Geneviève LD, Martani A, Wangmo T, Paolotti D, Koppeschaar C, Kjelsø C, et al. Participatory Disease Surveillance Systems: Ethical Framework. J Med Internet Res 2019;21(5):e12273.

9. Kalkman S, Mostert M, Gerlinger C, van Delden JJM, van Thiel GJMW. Responsible data sharing in international health research: a systematic review of principles and norms. BMC Med Ethics 2019;20(1):21

10. Mascalzoni D, Bentzen HB, Budin-Ljøsne I, Bygrave LA, Bell J, Dove ES, et al. Are Requirements to Deposit Data in Research Repositories Compatible With the European Union's General Data Protection Regulation? Ann Intern Med 2019;170(5):332-4.

11. Krutzinna J, Taddeo M, Floridi L. Enabling Posthumous Medical Data Donation: An Appeal for the Ethical Utilisation of Personal Health Data. Sci Eng Ethics 2019;25(5):1357-87.

12. Parasidis E, Pike E, McGraw D. A Belmont Report for Health Data. N Engl J Med 2019;380(16):1493-5.

13. Chevrier R, Foufi V, Gaudet-Blavignac C, Robert A, Lovis C. Use and Understanding of Anonymiza- tion and De-Identification in the Biomedical Literature: Scoping Review. J Med Internet Res 2019;21(5):e13484.

14. Schairer CE, Cheung C, Kseniya Rubanovich C, Cho M, Cranor LF, Bloss CS. Disposition toward privacy and information disclosure in the context of emerging health technologies. J Am Med Inform Assoc 2019;26(7):610-9.

15. Bentzen HB, Høstmælingen N. Balancing Protection and Free Movement of Personal Data: The New European Union General Data Protection Regulation. Ann Intern Med 2019;170(5):335-7.

16. Oxman AD, Paulsen EJ. Who can you trust? A review of free online sources of "trustworthy" information about treatment effects for patients and the public. BMC Med Inform Decis Mak 2019;19(1):35.

17. Chiauzzi E, Wicks P. Digital Trespass: Ethical and Terms-of-Use Violations by Researchers Accessing Data From an Online Patient Community. J Med Internet Res 2019;21(2):e11985.

18. Pisani AR, Kanuri N, Filbin B, Gallo C, Gould M, Soleymani Lehmann L, et al. Protecting User Privacy and Rights in Academic Data-Sharing Partnerships: Principles From a Pilot Program at Crisis Text Line. J Med Internet Res 2019;21(1):e11507.

19. Anderson M, Anderson SL. How Should AI Be Developed, Validated, and Implemented in Patient
Care? AMA J ethics 2019;21(2):E125-130.

20. Reddy S, Allan S, Coghlan S, Cooper P. A governance model for the application of $\mathrm{AI}$ in health care. J Am Med Inform Assoc 2020;27(3):491-7.

21. Powell J. Trust Me, I'm a Chatbot: How Artificial Intelligence in Health Care Fails the Turing Test. J Med Internet Res 2019;21(10):e16222.

22. Fiske A, Henningsen P, Buyx A. Your Robot Therapist Will See You Now: Ethical Implications of Embodied Artificial Intelligence in Psychiatry, Psychology, and Psychotherapy. J Med Internet Res 2019;21(5):e13216.

23. Antonio MG, Petrovskaya O, Lau F. Is research on patient portals attuned to health equity? A scoping review. J Am Med Inform Assoc 2019;26(89):871-83.

Correspondence to:

Carolyn Petersen, MS, MBI, FAMIA

Mayo Clinic

200 First St. SW

Rochester, MN, 55905

USA

E-mail: petersen.carolyn@mayo.edu 
Appendix: Content Summaries of Selected Best Papers for the 2020 IMIA Yearbook, Special Section on Ethics in Health Informatics

\author{
Antonio MG, Petrovskaya 0, Lau F \\ Is research on patient portals attuned to \\ health equity? A scoping review
}

\section{J Am Med Inform Assoc 2019 Aug 1;26(8- 9):871-83}

In this scoping review, the authors assessed how research on patient portals addresses health inequity. They sought to understand the health equity concepts explicitly and implicitly addressed in patient portal research; identify gaps in such research; assess whether eHealth-related inequities are acknowledged in patient portal research; and identify strategies to reduce health inequities that are being tested in such research. The authors used the eHealth Equity Framework (eHEF) to identify search terms and searched CINAHL, MEDLINE, Embase, and Scopus for "patient portal" plus various health equity terms (e.g., socioeconomic factors, digital divide) to identify articles to include in the review. They then independently reviewed the 65 articles meeting the inclusion criteria. Using the eHEF for analysis, they identified four themes: 1) eHealth policies, governance approaches, and cultural and societal values may further inequities; 2) providers and patients differ in preferences for portal use based on social position; 3 ) diverse user-centered designs facilitate equitable portal implementation; and 4) intermediary strategies for promoting portal use among populations are frequently suggested. The authors note that published work focuses on barriers to portal use, which shifts responsibility for addressing barriers to those who already experience the largest health disparities and potentially obfuscates the effect of social, technical, economic, and political factors on outcomes. The authors conclude that the informatics community must focus on developing equitable strategies at the policy, practice, research, and implementation levels to drive change.

\section{Lehmann CU, Petersen C, Bhatia H, Berner ES, Goodman KW}

\section{Advance directives and code status information exchange: a consensus proposal for a minimum set of attributes \\ Camb Q Healthc Ethics 2019 Jan;28(1):178-85}

Advance directives (ADs) benefit patients and their families by improving care and quality of life, and by making it more likely that patients have the end-of-life experience they desire. However, the use of ADs and the communication of code status happen infrequently, and documenting ADs and code status in the electronic health record (EHR) remains difficult. Members of the American Medical Informatics Association's Ethics Committee determined that a minimum data set for the storage and exchange of code status information could support greater use of ADs, and they performed an environmental scan to identify existing resources that could facilitate such documentation in the EHR. Through multiple conference calls, work group members achieved consensus around a proposed minimum data set with links to the HL7 C_CDA Advance Directives Module. Data categories include information about: 1) the organization obtaining the code status information; 2) the patient; 3) supporting documentation; and 4) the desired code status information including mandatory, optional, and conditional elements. These three types of elements prevent the creation of an incomplete document that will not support achievement of patients' goals endof-life while managing the clinical burden associated with creating such documentation. The resulting data set facilitates communication of patient goals and preferences across multiple providers and health care settings. It is intended that the identified data elements function as a starting point for discussion among informaticians, physicians and staff, and EHR vendors.
Pisani AR, Kanuri N, Filbin B, Gallo C, Gould M, Lehmann LS, Levine R, Marcotte JE, Pascal B, Rousseau D, Turner S, Yen S, Ranney ML

Protecting user privacy and rights in academic data-sharing partnerships: principles from a pilot program at crisis text line

\section{J Med Internet Res 2019 Jan} 17;21(1):el1507

Collaborations between academic researchers and technology companies historically have been difficult to develop because of differing needs and goals related to data privacy and security, intellectual property, technical requirements, ethics, and other issues. Companies and academic institutions often are subject to different regulatory requirements, and companies may incur costs from sharing data for noncommercial use without gaining commensurate benefits from such activity, making companies reluctant to do so. This paper describes an 18-month pilot undertaken by a non-for-profit technology company with 20 research teams at 18 universities in which data from a crisis text line was shared for research purposes. Design, development, and implementation of principles and protocols for ethical, secure sharing of crisis text line user data were the main objectives of the work. To accomplish this, the company created a data ethics committee, identified policy barriers and potential ways to address them, publicized the initiative, revised the policy, and launched the pilot. After program completion, the company evaluated it against other potential program models and modified its approach as appropriate. This paper describes the resulting 3-step set of guidelines for working with academic research organizations, which focus on 1) define the value and suitability of data and institutions for data-sharing programs; 2) choose a model for collaboration involving data sharing; and 3 ) identify the most appropriate institutional structure and develop technical approaches for ethical, secure data sharing. The paper also describes how internal evaluation of the pilot indicated successful achievement of its primary goal, shares principles and processes that may be useful to other companies, and suggests other data-sharing models that may work better in other circumstances. 